\title{
Abordaje moderno del hiperparatiroidismo primario
}

\author{
Modern management of primary hyperparathyroidism
}

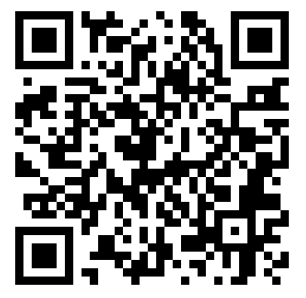

Recibido $18 / 01 / 2021$

\author{
${ }^{1}$ Dra. Maribel Baraquiso Pazos \\ Investigador independiente, San José, Costa Rica \\ (iD) https://orcid.org/0000-0002-1337-3696 \\ ${ }^{2}$ Dr. Alejandro José Corella Solano \\ Investigador independiente, San José, Costa Rica \\ (D) https://orcid.org/0000-0001-5784-2872
}

$\begin{array}{lr}\text { Corregido } & \text { Aceptado } \\ 27 / 01 / 2021 & 02 / 02 / 2021\end{array}$

\section{RESUMEN}

El hiperparatiroidismo primario representa el tercer padecimiento endocrinológico más frecuente en la actualidad. La mayoría de los casos cursa de manera asintomática, por lo que es una patología infradiagnosticada. Existen tres etiologías principales de hiperparatiroidismo primario: el adenoma, la hiperplasia y el carcinoma paratiroideo. Los métodos diagnósticos van desde estudios de laboratorio hasta métodos de imagen como ultrasonido, cintigrama, tomografía axial computarizada y resonancia magnética. El tratamiento de estas entidades se basa principalmente en la resección de las glándulas paratiroideas.

PALABRAS CLAVE: endocrinología; glándulas paratiroides; hiperparatiroidismo; hipercalcemia.

\section{ABSTRACT}

Primary hyperparathyroidism represents the third most common endocrine disease nowadays. Since most of the cases are asymptomatic, this remains as an underdiagnosed pathology. There are three main etiologies of primary hyperparathyroidism: adenoma, hyperplasia, and parathyroid carcinoma. Diagnostic methods range from laboratory studies to imaging methods such as ultrasound, scintigraphy, computerized axial tomography, and magnetic resonance. The treatment of these entities is based mainly on the resection of the parathyroid glands.

KEYWORDS: endocrinology; parathyroid glands; hyperparathyroidism; hypercalcemia.

\footnotetext{
${ }^{1}$ Médica general, graduada de la Universidad de Ciencias Médicas (UCIMED), cód. MED16657. Correo: mabipazos@hotmail.com

${ }^{2}$ Médico general, graduado de la Universidad de Ciencias Médicas (UCIMED). cód. MED16577. Correo: acorella96@gmail.com
} 


\section{INTRODUCCIÓN}

Las glándulas son pequeñas estructuras localizadas posteriormente a la glándula tiroidea (1-5). La principal secreción de estas glándulas es la hormona paratiroidea (PTH), la cual se encarga de la homeostasis del calcio sérico en el ser humano $(3,6,7)$. El hiperparatiroidismo primario representa el tercer desorden endocrinológico más frecuente en la actualidad (8-10).

Las glándulas paratiroides fueron descritas por primera vez por el anatomista, de nacionalidad inglesa, Sir Richard Oven, en el año 1852.

Dicho hallazgo se dio mediante la realización de una autopsia a rinocerontes del Zoológico de Londres. Asimismo, en 1880 el estudiante de medicina Ivar Sandtrom reportó la presencia de glándulas adyacentes al tejido tiroideo en los seres humanos, de allí su nombre glándulas paratiroideas.

Por otra parte, Olsh fue el primer cirujano que realizó una paratiroidectomía en un paciente con un adenoma paratiroideo, en Estados Unidos, en el año 1928 en el Hospital de la Universidad de Washington $(2,3,11)$.

El hiperparatiroidismo primario en la mayoría de los casos se presenta de manera asintomática; sin embargo, puede tener manifestaciones clínicas diversas que pueden afectar la calidad de vida del paciente y aumentar su morbi-mortalidad $(7,9,12,13)$.

El objetivo de esta publicación se basa en brindar a los médicos generales información acerca de la patogénesis y el diagnóstico de pacientes con hiperparatiroidismo primario, para de esta forma lograr un abordaje moderno y preciso que disminuya la morbilidad de estos.

\section{MÉTODO}

Para la realización de este artículo se hizo una búsqueda en las bases de datos electrónicas PubMed, Elsevier y Uptodate, con las frases "fisiología paratiroidea", "hiperparatiroidismo primario", "glándulas paratiroideas", "adenoma paratiroideo", "carcinoma paratiroideo" e "hiperplasia paratiroidea". Se incluyeron revisiones de tema y publicaciones originales creadas entre el período 2016 - 2021. Se analizaron documentos en idiomas español e inglés. En total se recopilaron 15 artículos para esta revisión y 3 libros de texto.

\section{ANATOMÍA DE LAS GLÁNDULAS PARATIROIDES}

Las glándulas paratiroides usualmente son pequeñas, con un peso aproximado entre 30 - 50 mg (1-5). Su coloración es amarillenta o cafezusca, pero puede cambiar con la edad del paciente: más oscura en jóvenes y más clara en mayores, esto por la cantidad de grasa que aumenta progresivamente con la edad (2-4).

Los seres humanos cuentan con cuatro glándulas paratiroideas, dos superiores y dos inferiores; pero incluso se han visto pacientes hasta con doce glándulas supernumerarias aunque solamente un $0.7 \%$ de ellas representan la causa de la enfermedad (2).

Estas glándulas se encuentran situadas posteriormente a la glándula tiroidea (figura 1), con una relación sumamente importante con el nervio laríngeo recurrente (NLR); así pues, las glándulas superiores se encuentran usualmente profundas al NLR y las inferiores se encuentran ventrales a este $(2,4)$. 
Las glándulas paratiroideas superiores se asocian con la cápsula posterior de la tiroides y están cubiertas por una extensión de la fascia pretraqueal; estas estructuras al agrandarse pueden ubicarse en el espacio retro o paraesofágico. Por otra parte, las paratiroideas inferiores pueden encontrarse en ubicaciones más variables debido a su embriología, ya que migran desde el saco faríngeo junto con el timo. Usualmente se encuentran cerca del polo inferior de la glándula tiroidea (1-4).

La vascularización de las glándulas paratiroideas superiores e inferiores se da mayoritariamente por la arteria tiroidea inferior, rama del tronco tirocervical $(2,9)$. Hasta un $10 \%$ de las glándulas paratiroideas inferiores son irrigadas por la arteria tiroidea superior (2). El drenaje venoso se da a través de las vénulas tiroideas superior, media e inferior; y el drenaje linfático por parte de los nódulos pretraqueales, prelaríngeos y yugulodigástricos $(3,9)$.

Figura 1. Localización de las glándulas paratiroideas (vista posterior)

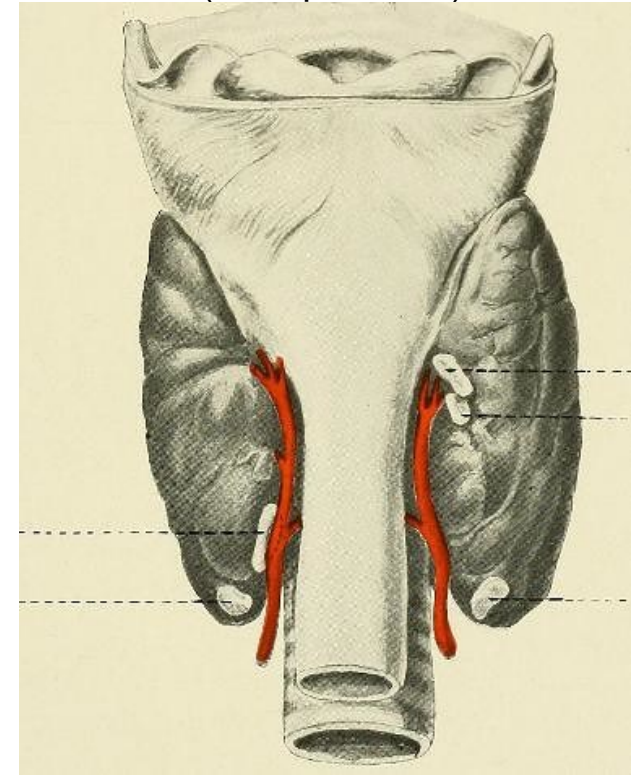

Fuente. Imagen de dominio público. Autor: Ochsner, Albert J. Tomada de:

https://search.creativecommons.org/photos/53acbc c9-4fd5-4ff3-828e-df0eb69dfcb5

\section{FISIOLOGÍA DE LAS GLÁNDULAS} PARATIROIDEAS

- La PTH es el producto secretado por las células principales de las glándulas paratiroides en respuesta a las variaciones séricas de calcio $(3,6,7)$. El gen de la PTH se localiza en el cromosoma 11. Se sintetiza inicialmente una pre-pro-hormona de 115 aminoácidos que se procesa para formar la PTH, una proteína de 84 aminoácidos (6). Esta hormona se concentra en gránulos y vesículas de las células principales antes de secretarse Una vez secretada tiene una vida media aproximada de 2 minutos, con un $70 \%$ de degradación hepática y $20 \%$ renal (7). Actúa sobre el PTH/PTHrP tipo 1 (o PTH1R), un receptor acoplado a proteína $G$, encontrado principalmente en hueso y riñón (6).

- El principal regulador de la secreción de la PTH es el calcio sérico. El calcio de líquido extracelular (LEC) se une al receptor sensor de calcio (CaSR), una proteína $G$ capaz de activar la fosfolipasa C y bloquear la producción de AMP cíclico, inhibiendo la secreción de PTH, degradando la PTH bioactiva dentro de los gránulos y vesículas, e inhibiendo la transcripción de su gen. En circunstancias de normocalcemia, la biosíntesis de PTH y su secreción son mínimas. Otros reguladores de la PTH son: la 1,25(OH)2D3 (forma activa de la vitamina $\mathrm{D}$ ) que suprime la transcripción de la PTH; la elevación sérica de fosfato inorgánico que aumenta la secreción de PTH directamente y por medio de la disminución del calcio sérico. Factores como el litio y el FGF23 también regulan la secreción de la PTH. $(6,7)$. 
- El número de células paratiroideas aumenta durante la infancia, pero varía poco en la adultez. Puede haber un incremento en su número en situaciones crónicas de hipocalcemia, niveles bajos de 1,25(OH)2D3, hiperfosfatemia, uremia o por crecimiento neoplásico (7). Entre las acciones de la $\mathrm{PTH}$ se encuentran en $(6,7,10)$ :

- Riñón: estimulación de la reabsorción del calcio (principalmente en el túbulo contorneado distal por los canales TRPV5 y TRPV6), inhibición de la reabsorción de fosfato (a nivel tubular proximal y distal, al bloquear los transportadores sodio-fosfato), transcripción de la 1-alfahidroxilasa (enzima encargada de la transformación de 25(OH)D a 1,25(OH)2D3), inactivación de la enzima 24-hidroxilasa (se encarga de inactivar la vitamina D), inhibe la reabsorción de agua, sodio y bicarbonato en el túbulo proximal.

- Hueso: promueve la diferenciación de los osteoblastos para la mineralización ósea. Sin embargo, los osteoblastos $y$ osteocitos estimulados por la PTH incrementan la producción de la citokina ligando de receptor activador para el factor nuclear $\mathrm{k}$ $B$ (RANKL), que se une al receptor activador para el factor nuclear $\mathrm{K}$ B (RANK) en el osteoclasto para promover su diferenciación, por lo tanto, la resorción ósea. El balance entre estos efectos depende del nivel de exposición del hueso a la PTH: de manera sostenida o continua, los efectos catabólicos (resorción ósea) predominan, mientras que, de manera intermitente o discontinua, los efectos anabólicos (formación ósea) predominan.

\section{DEFINICIÓN Y EPIDEMIOLOGÍA}

El término hiperparatiroidismo se refiere a la presencia de niveles circulantes altos de PTH $(3,9,10)$. Se habla de una etiología primaria cuando es un trastorno propio de las paratiroides que ocasiona esta alteración (7). El hiperparatiroidismo primario es el tercer desorden endocrino más frecuente, después de la diabetes mellitus y los trastornos tiroideos, y es la causa más frecuente de hipercalcemia en pacientes ambulatorios. Conocer su verdadera prevalencia e incidencia es complicado, ya que la mayoría de los casos cursan asintomáticos y no se detectan. Suelen afectar entre 2 y 3 veces más a las mujeres que a los hombres (8-10).

\section{MANIFESTACIONES CLÍNICAS}

- Hiperparatiroidismo primario clásico: se refiere a la enfermedad sintomática multisistémica. Hoy en día es poco común en sistemas de salud desarrollados (12).

- Manifestaciones óseas: se deben a un incremento marcado de la resorción ósea por medio de los osteoclastos. Esta enfermedad ósea fue descrita por Von Recklinghausen como "osteítis fibrosa quística". Dentro 
de los hallazgos se pueden encontrar: desmineralización generalizada, resorción subperióstica (más evidente en las falanges de las manos), quistes óseos, osteoclastomas (tumores café por unión de varios osteoclastos multinucleados), fracturas patológicas (por ejemplo en huesos largos 0 costillas), moteado fino en sal y pimienta a nivel del cráneo, pérdida de la diferenciación entre tabla externa e interna del cráneo, dolor óseo, curvamiento de los hombros, cifosis, pérdida de estatura por fracturas vertebrales, entre otros $(7,10)$.

- Manifestaciones renales y urinarias: se dan por el exceso de calcio en este sistema secundario a la hipercalcemia y mayor reabsorción tubular. Se pueden hallar: nefrolitiasis a repetición, nefrocalcinosis, poliuria (por la calciuria), polidipsia (por la deshidratación) y anormalidades en la función renal $(7,10,12)$.

- Otras manifestaciones: anorexia, náuseas y vómitos, constipación, dolor abdominal, úlcera péptica, pancreatitis aguda y crónica, debilidad muscular proximal, alteraciones psiquiátricas (apatía, depresión, fatiga). Estas son causadas por la hipercalcemia secundaria al exceso de PTH $(7,10)$.

- Hiperparatiroidismo primario asintomático: actualmente esta es la presentación de más del $80 \%$ de los pacientes. Las distintas bibliografías definen los casos asintomáticos como aquellos donde no existen manifestaciones óseas y renales clínicamente evidentes; por lo que el diagnóstico se realiza de forma incidental al encontrar un calcio sérico elevado. Sin embargo, a pesar de llamarse asintomático, se ha demostrado que estos pacientes si tienen algunas alteraciones clínicas por este estado patológico $(7,9,12,13)$.

- Perfil bioquímico: hipercalcemia (por lo general menos de $1 \mathrm{mg} / \mathrm{dL}$ sobre el límite superior), fosfato en la mitad inferior del rango normal, 25(OH)D en rangos insuficientes o deficientes con $1,25(\mathrm{OH}) 2 \mathrm{D} 3$ en rangos normales-altos (12). Estas leves alteraciones suelen mantenerse estables por años (13).

- Manifestaciones óseas: las densitometrías óseas muestran un catabolismo de la porción cortical del hueso, por ejemplo, en el tercio distal del antebrazo, mientras que a nivel vertebral hay menos alteraciones, aunque paradójicamente hay un aumento de las fracturas vertebrales. La prevalencia de osteoporosis en esta entidad varía entre estudios $(7,10,12)$.

- Manifestaciones renales: la nefrolitiasis hoy en día se presenta en menos de una cuarta parte de los pacientes con hiperparatiroidismo primario, aunque puede haber afección de 
la función renal hasta en una tercera parte de los asintomáticos. Esta alteración es poco progresiva en el tiempo (7, 12).

\section{- Manifestaciones}

neuropsiquiáticas: se ha descrito depresión, ansiedad, fatiga, disminución de la calidad de vida, alteraciones del sueño y disfunción cognitiva $(7,12,13)$. Dichas manifestaciones se le han achacado a la hipercalcemia, aunque su reversibilidad con el tratamiento adecuado no es congruente en diversos estudios (12).

\section{- Manifestaciones}

cardiovasculares: se asocia el hiperparatiroidismo primario con hipertensión arterial, hipertrofia del ventrículo izquierdo y calcificación valvular $(7,12,13)$. Existe controversia si estas consecuencias se deben al hiperparatiroidismo o si es debido a factores de riesgo tradicionales, ya que hay heterogeneidad entre estudios sobre la reversibilidad de dichas condiciones después del tratamiento (12).

- Hiperparatiroidismo

primario normocalcémico: tiene una prevalencia del $3 \%$ o menor. Los niveles de calcio se encuentran dentro de los rangos normales, y los cambios bioquímicos son más sutiles. Al igual que en los casos asintomáticos, tienen tasas más altas de osteoporosis, fracturas y nefrolitiasis, y de hecho se suele diagnosticar a partir de un evento como estos (12).

\section{PRINCIPALES ETIOLOGÍAS}

Las principales etiologías de un hiperparatiroidismo primario son el adenoma, la hiperplasia y el carcinoma paratiroideo, cuyas características se citarán a continuación.

- Adenoma de paratiroides: son responsables de aproximadamente el 85\% de los casos de hiperparatiroidismo primario. El pico de presentación suele ser entre la $5^{\text {ta }}$ y la $6^{\text {ta }}$ década de vida, es más frecuente en el sexo femenino y afecta más a las glándulas paratiroides inferiores $(1,14)$. Suelen ser neoplasias redondas, bien circunscritas, que en pocas ocasiones llegan a ser grandes. Histológicamente están formadas por células principales, suelen tener una cápsula fibrosa, con poco contenido de grasa, y los tumores de mayor tamaño pueden tener áreas de fibrosis, de hemorragia y de degeneración quística. Tienen una actividad mitótica baja (1). El origen de los adenomas paratiroideos se debe a mutaciones monoclonales que ocasionan anormalidades en la respuesta al calcio, en los cuales se necesitan niveles más altos de calcio para suprimir la PTH (7). En algunos de los casos esporádicos, se han implicado las mutaciones inactivadoras del gen MEN1, que codifica la transcripción de menin, un supresor tumoral, el cual también está alterado en las neoplasias endocrinas múltiples tipo 1 (NEM1). Además, el proto-oncogen Cyclin D1/PRAD1, también se ha relacionado con la génesis de esta neoplasia. Como todo padecimiento debido a mutaciones del ADN, es más frecuente en personas que recibieron altas dosis de radiación ionizante a nivel de cuello $(1,7,10)$. En los 
casos familiares, se asocia ampliamente con el NEM1, en el cual el $95 \%$ de los pacientes tendrán adenomas paratiroideos, los cuales ocurren de manera asimétrica y asincrónica, y de manera mucho menos frecuente con el NEM2a, síndrome que se debe a mutaciones del gen RET $(1,7)$.

Se le llama adenoma atípico a aquel que histológicamente tiene características sospechosas de malignidad, sin embargo, no infiltra a su cápsula, el tejido, los vasos o los nervios adyacentes. Se suelen manejar igual que los adenomas típicos (1). En estos pacientes se ha identificado la mutación del gen CDC73/HRPT2, que codifica para la parafibromina y puede causar un síndrome de Hiperparatiroidismo-tumor mandibular. En ocasiones esta última mutación se asocia a carcinoma de paratiroides $(1,7,10)$.

La gran mayoría de los pacientes con este tipo de neoplasias se presentan como un hiperparatiroidismo primario asintomático, como descrito anteriormente (14).

- Diagnóstico: en la actualidad se utilizan 4 métodos de imagen para localizar el adenoma paratiroideo: $(7,9,13)$

\section{Ultrasonido}

(US): usualmente las glándulas paratiroideas son invisibles bajo este método; sin embargo, cuando existe un adenoma se pueden observar como imágenes hipoecoicas ovaladas, redondas 0 lobuladas con una cápsula ecogénica bien definida. Usualmente los adenomas presentan un anillo de vascularización al utilizar US de tipo Doppler, diferencia de nódulos linfáticos. Además, con este tipo de US se puede observar un aumento en la vascularización del lóbulo tiroideo ipsilateral.

2. Cintigrama de paratiroides con Tecnesio-99m

SESTAMIBI: se observa un aumento de la captación y retención del Tecnesio-99m. Puede causar falsos positivos con otras patologías tales como carcinoma de paratiroides, nódulos o malignidades tiroideos; por lo que se debe correlacionar su uso con otras técnicas de imagen.

3. Tomografía multifase paratiroidea en $4^{\text {ta }}$ dimensión (TAC-4D): se observa un nódulo de tejido blando con realce en la fase arterial y disminución o ausencia en la fase venosa. Esta técnica sin embargo cuenta con la limitación de la gran cantidad de radiación a la que se expone el paciente.

4. Resonancia magnética (RM): se observará una mayor señal en realce con $\mathrm{T} 2$, con apariencia homogénea en su mayoría.

Cabe recalcar que se deben utilizar dos o más métodos de imagen para lograr una localización adecuada del 
adenoma de forma preoperatorio y con esto garantizar la posibilidad de una paratiroidectomía mínimamente invasiva (7).

- Tratamiento quirúrgico: la paratiroidectomía, o resección de las glándulas paratiroideas, se considera el tratamiento definitivo para los pacientes con hiperparatiroidismo primario causados por adenomas en pacientes sintomáticos $(9,13)$.

Cabe recalcar que al remover una glándula paratiroidea debe haber un descenso a los 10 minutos de mínimo $50 \%$ de los niveles de PTH $(7,13)$.

En la tabla 1 se enumeran las guías para la realización de una paratiroidectomía en aquellos pacientes asintomáticos $(2,9,11,13)$.

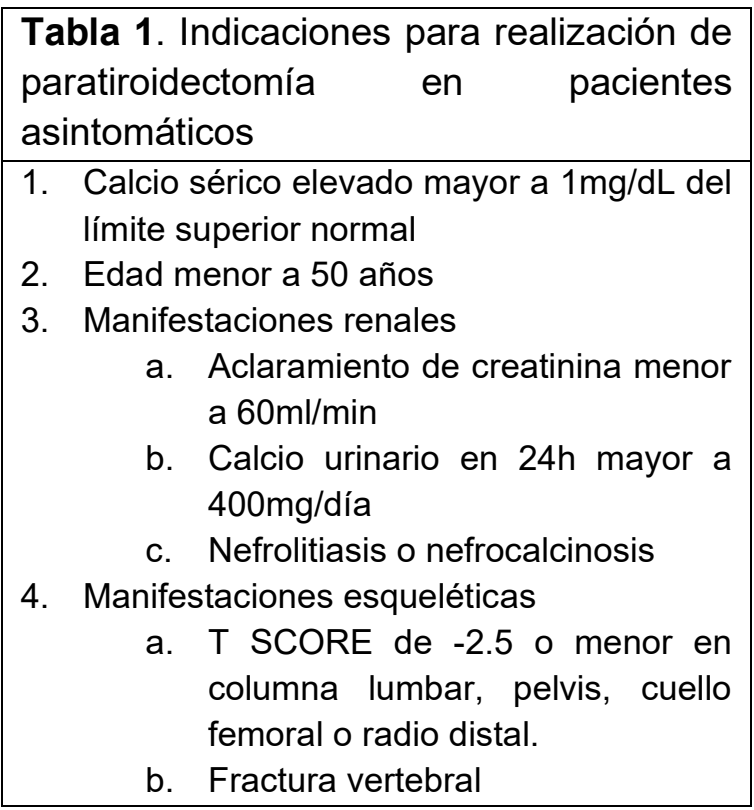

- Tratamiento médico: en todos los pacientes que no se sometan a cirugía se les debe de dar un seguimiento estricto anual con mediciones de: calcio sérico, PTH, creatinina sérica y cálculo de la tasa de filtración glomerular. Además, se debe de realizar una densitometría ósea cada 1 o 2 años $\quad(7,10,15)$ Como recomendaciones generales se les indica mantener una adecuada hidratación, evitar diuréticos y encamamientos prolongados, y buscar atención médica en cuadros de vómitos o diarrea abundante. Estas medidas son dadas con el fin de evitar un empeoramiento de la hipercalcemia. Es importante añadir que no se hace restricción dietética de calcio (7). Actualmente, no existe un tratamiento médico definitivo. Se han utilizado bifosfonatos (como el Alendronato intravenoso) o Raloxifeno en mujeres postmenopáusicas, que tienen resultados positivos a nivel óseo, pero no tienen efectos significativos ni persistentes en los valores de calcio sérico ni de PTH. De manera alternativa se usan agentes calcimiméticos, es decir, que activan el CaSR en la glándula paratiroides para disminuir la producción de PTH. El Cinacalcet es el único calcimimético aprobado por la Food And Drug Administration (FDA), y solamente está indicado para el hiperparatiroidismo secundario en pacientes con enfermedad renal crónica en diálisis. Este medicamento se ha utilizado en hiperparatiroidismo 
primario, con resultados beneficiosos en algunos de los pacientes con cuadros sintomáticos, y poco éxito en los casos asintomáticos $(7,10)$.

- Hiperplasia de paratiroides: la hiperplasia de las paratiroides se debe al aumento en la masa celular de la glándula debido a la proliferación celular. En más del $50 \%$ de los casos este crecimiento es simétrico, es decir, de todas las paratiroides. La hiperplasia secundaria es mucho más frecuente que la primaria $(1,7)$. La hiperplasia primaria de las paratiroides es la segunda causa más frecuente de hiperparatiroidismo primario, con una prevalencia del 10 al $15 \%(10,14)$. En los casos esporádicos, se desconoce la causa y se considera que se debe a un crecimiento policlonal. Los casos familiares se presentan en pacientes con NEM1, NEM2a y el síndrome de Hiperparatiroidismo-tumor mandibular $(7,10)$.

- Diagnóstico y manejo: es muy importante la distinción entre adenoma e hiperplasia paratiroidea, ya que con la remoción la glándula afectada se puede curar el padecimiento dado por un adenoma, contrario a los casos de hiperplasia, donde se necesita quitar más tejido paratiroideo para tratar el padecimiento (7). Se sospecha hiperplasia en los pacientes que, con una hipercalcemia modesta, el US y el cintigrama con Tecnesio-99m son negativos. También se sospecha cuando se detecta un bajo peso de las glándulas de manera intraoperatoria (16). Pacientes con hiperparatiroidismo primario menores de 30 años también son sospechosos por hiperplasia glandular. Actualmente la única manera confiable de distinguir ambas entidades es mediante el estudio patológico, principalmente observando el crecimiento de tamaño de la paratiroides, la cantidad y la distribución grasa de la glándula (7).

Todos los pacientes en los que se sospeche enfermedad multiglandular y en los conocidos con NEM1, se debe realizar una exploración quirúrgica bilateral del cuello $(7,16)$. Los estudios de localización de cintigrama con Tecnesio-99m tienen poca sensibilidad y bajo valor predictivo positivo, por lo cual siempre se complementa con US o TAC-4D. Además, a la hora de hacer el muestreo de PTH 10 minutos post-resección de la o las glándulas que se consideren afectadas, se espera una caída del $90 \%$ de la PTH sobre su valor basal o su retorno a valores normales para considerar la resección exitosa (7). En estos pacientes se puede realizar una resección limitada de las glándulas visiblemente anormales, una paratiroidectomía subtotal con criopreservación de tejido, o una paratiroidectomía total con autotrasplante inmediato al antebrazo $(7,10,16)$. 
- Carcinoma de paratiroides: este tipo de neoplasia maligna tiene una prevalencia aproximada de $0.005 \%$ de todos los cánceres que afectan al ser humano y representa menos del $1 \%$ del total de condiciones que causan hiperparatiroidismo primario $(3,4,17,18)$. Su prevalencia es igual en mujeres y en hombres, con una edad media de aparición entre 45-59 años. $(17,18)$. Hasta la actualidad no se han encontrado factores predisponentes para padecer de esta enfermedad y su patogénesis es desconocida; sin embargo, puede aparecer de forma esporádica o ligada a un síndrome genético, como las NEM tipo 1 y tipo 20 el Síndrome de Hiperparatiroidimotumor mandibular $(17,18)$. Se han visto mutaciones genéticas del gen supresor tumoral CDC73, asociadas a la codificación de la parafibromina. Dicha alteración se encuentra hasta en el $70 \%$ de los pacientes con carcinomas paratiroideos esporádicos y también en el síndrome de hiperparatiroidismotumoración mandibular $(1,18)$.

Asimismo, factores como radioterapia previa en la región cervical o historia de neoplasias malignas previas pueden considerarse factores de riesgo para desarrollar esta patología $(17,18)$.

- Manifestaciones clínicas: los carcinomas de paratiroides generalmente presentan una sintomatología más severa que las otras enfermedades citadas anteriormente (3). Cabe recalcar que estos pacientes presentan una hipercalcemia severa (con sintomatología como úlceras pépticas, fatiga, cansancio, náuseas y pancreatitis) y un $90 \%$ de estas neoplasias se consideran hormonalmente funcionales $(3,17,18)$. Asimismo, pueden asociar signos neurológicos o disfonía por invasión del NLR (18).

A la exploración física, entre un $30-76 \%$ de los casos presentarán una masa palpable y un $15-30 \%$ de los pacientes tendrán metástasis en los ganglios linfáticos al momento de la presentación, con una prevalencia de metástasis a distancia de un $30 \%$, en lugares como el pulmón, hueso e hígado $(1,3,4,17,18)$.

- Diagnóstico: usualmente es una entidad de difícil diagnóstico, el cual si se realiza de forma tardía es incurable $(3,17)$. Los niveles de calcio sérico suelen ser mayores a $14 \mathrm{mg} / \mathrm{dl}$ y los de PTH pueden estar desde tres hasta diez veces por encima del valor normal. Asimismo, otros marcadores como la fosfatasa alcalina y la gonadotropina coriónica humana están elevadas en la mayoría de los casos $(1,4,17,18)$. Además, la presencia de una masa mayor a $3 \mathrm{~cm}$ en la glándula debe aumentar la sospecha de un carcinoma de paratiroides (18).

En el caso de sospecha de carcinoma se debería realizar una tomografía axial computarizada o RM para demostrar la invasión a estructuras locales y guiar el abordaje quirúrgico. No se recomienda el uso de biopsia por aspiración con aguja fina (BAAF) 
debido al riesgo de ruptura y diseminación tumoral $(4,17)$.

Es importante mencionar que el carcinoma paratiroideo es muy raramente sospechado preoperatoriamente y la mayoría de los diagnósticos son intraoperatorios (7).

○ Tratamiento:

la paratiroidectomía representa el tratamiento inicial más apropiado para estos pacientes. Se debe realizar una resección completa con márgenes microscópicos negativos para ofrecer el mayor porcentaje de curación. Es importante realizar una disección en bloque de los tejidos implicados sin comprometer la cápsula tumoral. Asimismo, se recomienda la disección del lóbulo tiroideo ipsilateral al carcinoma y el nivel linfático cervical VI ipsilateral. Si el tumor se encuentra unido al NLR se tomará la decisión de resecar el nervio con una posterior reconstrucción vía anastomosis o injerto del asa cervical. $(5,7,17,18)$.

El uso de radioterapia no posee evidencia actual como método inicial de tratamiento del carcinoma de la glándula paratiroidea. Sin embargo, se puede utilizar radioterapia adyuvante postoperatoria para disminuir la tasa de recurrencias y mejorar el control local de la tumoración $(17,18)$. Por otra parte, no se han demostrado resultados convencedores acerca del tratamiento con quimioterapia con agentes como doxorrubicina, bleomicina o carboplatino; ya que no se ha visto una mejoría en la hipercalcemia o en las tasas de supervivencia $(17,18)$.

La supervivencia de estos pacientes ronda el $66-80 \%$ a los 10 años, con una tasa de recurrencias de entre $40-63 \%$ $(17,18)$.

\section{CONCLUSIONES}

El hiperparatiroidismo primario es una patología frecuente de la consulta endocrinológica, que debe ser abordado de manera precisa para poder lograr un diagnóstico certero. Se debe tomar en cuenta que la gran mayoría de los pacientes serán asintomáticos; sin embargo, se debe prestar atención a manifestaciones renales y óseas que puedan pasar desapercibidas tanto por el paciente como por el médico tratante. A pesar de que la mayoría de los pacientes con hiperparatiroidismo primario presentarán un adenoma como causa de su patología, es de relevancia realizar diagnóstico diferencial con hiperplasia o carcinoma paratiroideo.

La combinación de estudios de imagen es la manera más adecuada para lograr identificar cuál de las glándulas paratiroideas se encuentra afectada, además de proporcionar un mejor campo de trabajo para el cirujano y lograr hacer una cirugía mínimamente invasiva en el paciente que lleve a menos complicaciones con una consecuente mejoría de la morbi-mortalidad del paciente. Asimismo, en aquellos pacientes asintomáticos que no cumplan criterios de cirugía deben manejarse de manera médica para reducir las 
complicaciones que pueden causar los niveles elevados de calcio en los seres humanos.

\section{REFERENCIAS}

1. Guilmette J, Sadow PM. Parathyroid Pathology. Surg Pathol Clin. 2019; 12 (4): 1007-1019. https://doi.org/10.1016/j.path.2019.08.006

2. Reid LM, Kamani D, Randoplh GW. Management of Parathyroid Disorders. Cummings Otolaryngology Head and Neck Surgery. 7ma edición. Filadelfia, Pensilvania: Elsevier: 2020. 1899-1916.

3. Demir AD. A review of parathyroid mass and patients with nonspecific complaints. J Int Med Res. 2020; $0 \quad$ (0): $1-6$. https://doi.org/10.1177/0300060519827169

4. Jason DS, Balentine CJ. Intraoperative Decision Making in Parathyroid Surgery. Surg Clin North Am. 2019; $99 \quad$ (4): $\quad 681-691$. https://doi.org/10.1016/j.suc.2019.04.008

5. Baj J, Sitarz R, Łokaj M, Forma A, Czeczelewski $M$, Maani A, et al. Preoperative and Intraoperative Methods of Parathyroid Gland Localization and the Diagnosis of Parathyroid Adenomas. Molecules [Internet]. MDPI AG; 2020 Apr 9;25(7):1724. Available from: http://dx.doi.org/10.3390/molecules25071724

6. Goltzman D. Phisiology of Parathyroid Hormon. Endocrinol Metab Clin North Am. 2018; 47 (4): 743-758. https://doi.org/10.1016/j.ecl.2018.07.003

7. Bringhurst FR. Demay MB, Kronenberg HM. Hormones and Disorders of Mineral Metabolism. Williams Textbook of Endocrinology. 14va edición. Filadelfia, Pensilvania: Elsevier; 2020. 1196-1255.

8. Rao SD. Epidemiology of Parathyroid disorders. Best Pract Clin Endocrinol Metab. 2018; 32 (6): 773-780. https://doi.org/10.1016/j.beem.2018.12.003

9. Kuzminski S, Sosa JA, Hoang JK. Update in Parathyroid Imaging. Magn Reson Imaging Clin N Am. 2018; $26 \quad$ (1): 151-166. https://doi.org/10.1016/j.mric.2017.08.009

\section{Los autores declaran no tener conflicto de interés.}

10. Shoback DM, Schafer AL, Bikle DD. Metabolic Bone Disease. Greenspan's Basic and Clinical Endocrinology. 10ma edición. Nueva York, Estados Unidos: McGraw- Hill: 2017. 239-297.

11. Sethi N, England RJ. Parathyroid surgery:from inception to the modern day. $\mathrm{Br} \mathrm{J}$ Hosp Med (Lond). $\quad 2017 ; \quad 78 \quad$ (6): 333-337. https://doi.org/10.12968/hmed.2017.78.6.333

12. Walker MD, Silverberg SJ. Primary hyperparathyroidism. Nat Rev Endocrinol. 2018; $14 \quad(2)$ : 115-125. https://doi.org/10.1038/nrendo.2017.104

13. Insogna KL. Primary hyperparathyroidism. N Eng J Med. 2018; 379 (11): 1050-1059. https://doi.org/10.1056/NEJMcp1714213

14. Fuleihan GE, Arnold A. Pathogenesis and etiology of primary hyperparathyroidism. 2020. Uptodate. Disponible en: https://www-uptodatecom.binasss.idm.oclc.org/contents/pathogenesisand-etiology-of-primaryhyperparathyroidism?search=pathogenesis $\% 20$ a nd\%20etiology\%20of\%20primary\%20hyperparat hyroidism\&source=search result\&selectedTitle $=1$ $\sim$ 120\&usage type=default\&display rank=1

15. Zhu CY, Sturgeon C, Yeh MW. Diagnosis and Management of Primary Hyperparathyroidism. JAMA. 2020; $323 \quad$ (12): 1186-1187. https://doi.org/10.1001/jama.2020.0538

16. Mc Henry CR, Shi HH. Can parathyroid hyperplasia be predicted preoperatively?. Am J Surg. 2018; $215 \quad$ (3): 389-392. https://doi.org/10.1016/j.amjsurg.2017.10.051

17. Goswamy J, Lei M, Simo R. Parathyroid carcinoma. Curr Opin Otolaryngol Head Neck Surg. 2016; $24 \quad$ (2): $155-162$. https://doi.org/10.1097/MO0.0000000000000234

18. Cetani F, Pardi E, Marcocci C. Parathyroid Carcinoma. From Hom Res. 2019; 51: 63-76. https://doi.org/10.1159/000491039 